\title{
SMALLEST ENCLOSING BALL MULTIDISTANCE
}

\author{
I. AGUILÓ*, J. MARTÍN*, G. MAYOR*, J. SUÑER*†, AND O. VALERO*
}

\begin{abstract}
The smallest enclosing ball problem is analyzed in the class of proper metric spaces. By using the diameter of the smallest enclosing ball of a set of points, we find conditions in order to ensure that the mentioned measure is a multidistance.
\end{abstract}

Keywords. Metric space, Fermat multidistance, smallest enclosing ball, midpoint property, Fermat property, m-dimensional Euclidian space.

1. Introduction. The problem of finding the smallest enclosing ball (SEB) of a finite set of points is a well-studied one with a large number of applications in many social and engineering areas. The SEB problem was according some authors first mentioned by J.J. Sylvester in 1857 ([7]).

This problem can be described as: Given a finite set $P$ of points in the $m$ dimensional Euclidean space $R^{m}$, find a ball $B(c ; r)=\left\{x \in R^{m} ; d(x, c) \leq r\right\}$ (closed ball with center $c \in R^{m}$ and radius $r \geq 0$ ) such that:

i) $B(c ; r) \supset P$

ii) If $B\left(c^{\prime} ; r^{\prime}\right) \supset P$ then $r^{\prime} \geq r$

It can be easily proved that for any finite set $P$ there exists a unique ball $B(c ; r)$ satisfying the above conditions. We call it the smallest enclosing ball of $P$.

A theoretical analysis for the SEB problem is given in [2]. In particular, it is proved that the $k$-circumscribing enclosing ball with smallest $k$ is the smallest enclosing ball. Recall that, given a finite set of points $P$ in $R^{m}$, the $k$-circumscribing enclosing ball of $P$ is an enclosing ball $B^{m}(c ; r)$ of $P$ having a $k$-dimensional ball $B^{k}(c ; r)$ as a $k$-dimensional large circle (a $k$-dimensional ball in a $k$-dimensional affine subspace passing through $c$, with the same center $c$ and radius $r$ ), which is uniquely determined by $k+1$ points in $P$. This fundamental result in [2] reduces a possible large number of computations in the higher dimensional case.

On the other hand, N. Megiddo presented in 1984 the first algorithm that solves the smallest enclosing circle problem in linear time for fixed dimension ([5]). An interesting paper which generalizes the smallest enclosing ball problem can be found in [6]. In this paper, two problems are studied: given a finite number of nonempty closed subsets of a normed space, find a ball with the smallest radius that encloses all of the sets, and find a ball with the smallest radius that intersects all of the sets.

The conventional definition of distance over a space specifies properties that must be obeyed by any measure of how separated two points in this space are. However, one

\footnotetext{
${ }^{*}$ Dept. of Mathematics and Computer Science, University of the Balearic Islands, 07071-Palma de Mallorca. Spain.

${ }^{\dagger}$ Corresponding author. e-mail: jaume.sunyer@uib.es
} 
often wants to measure how separated the members of a collection of more than two elements are. The conventional way to do it is to combine the pairwise distance values for all pairs of elements in the collection into an aggregate measure, for example, the arithmetic mean. It is clear that the aggregation function should be chosen in such a way that the multi-argument distance function satisfies a group of axioms consistent with those of the ordinary distance functions.

In [4] the concept of multi-argument distance (multidistance, for short) was introduced, where the conventional definition of distance was extended to apply to collections of more than two elements. It can be directly incorporated into many domains where ad hoc combinations of pairwise metrics are currently used, with the advantage that it allows the general treatment of the problem by means of an axiomatic procedure. See for example [1], where multidistances are applied in the field of Social Choice Theory. Of course, this approach includes in particular the case of multidistances defined from the pairwise distances, but it is more general [3].

There exist other definitions for multidistances, or multimetrics. For example, the one in [8], which is more general because it allows to measure the distance between the points of an infinite set, but it is more restrictive in some sense: reduced to lists, multimetrics in there correspond to strong multidistances in [4]. On the other hand, symmetry is not an axiom in [8], and hence it cannot be avoided, preventing the definition of asymmetric multidistances.

The purposes of this paper are the following:

1) Extend the SEB problem to a broad class of metric spaces.

2) Use the diameter of the SEB of a set of points as a measure of how separated those points are.

3) Analyse under which conditions that measure can be considered as a multidistance.

4) Prove that, in the Euclidean space $R^{m}$, the diameter of the SEB of a set of points defines a multidistance.

The paper is organized as follows: In Section 2 the definition of multidistance and basic examples are given. Section 3 is the core of the paper, it contains the main definitions and results. Conclusions are presented at the end of the paper.

2. Preliminaries. We recall here some definitions, properties and examples related to multidistances. See [4] for more details.

Definition 1. A function $D: \bigcup_{n \geqslant 1} X^{n} \rightarrow[0, \infty)$ is a multidistance on a non empty set $X$ when the following properties hold, for all $n \geq 1$ and $x_{1}, \ldots, x_{n}, y \in X$ :

(1) $D\left(x_{1}, \ldots, x_{n}\right)=0$ if and only if $x_{i}=x_{j}$ for all $i, j=1, \ldots n$,

(2) $D\left(x_{1}, \ldots, x_{n}\right)=D\left(x_{\pi(1)}, \ldots, x_{\pi(n)}\right)$ for any permutation $\pi$ of $1, \ldots, n$,

(3) $D\left(x_{1}, \ldots, x_{n}\right) \leqslant D\left(x_{1}, y\right)+\ldots+D\left(x_{n}, y\right)$. 
REMARK 1. If $D$ is a multidistance on $X$, then the restriction of $D$ to $X^{2},\left.D\right|_{X^{2}}$, is an ordinary distance on $X$. On the other hand, ordinary distances $d$ on $X$ can be extended in order to obtain multidistances.

EXAMPLE 1. The following are multidistances which can be defined on any metric space $(X, d)$. Observe that all of them extend the ordinary distance $d$.

- The maximum multidistance:

$$
D_{M}\left(x_{1}, \ldots, x_{n}\right)=\max _{i, j: 1, \ldots, n}\left\{d\left(x_{i}, x_{j}\right)\right\} .
$$

- The Fermat multidistance:

$$
D_{F}\left(x_{1}, \ldots, x_{n}\right)=\inf _{x \in X}\left\{\sum_{i=1}^{n} d\left(x_{i}, x\right)\right\} .
$$

$D_{F}$ is maximum in the sense that any other multidistance $D$ such that $\left.D\right|_{X^{2}}=$ $d$ takes values not greater than $D_{F}$, i.e. $D\left(x_{1}, \ldots, x_{n}\right) \leqslant D_{F}\left(x_{1}, \ldots, x_{n}\right)$, for all $\left(x_{1}, \ldots, x_{n}\right) \in X^{n}$.

Definition 2. A metric space $(X, d)$ is called proper if all bounded, closed balls are compact sets.

If $X$ is a proper metric space, then the infimum in (2) converts into a minimum.

\section{The Multidistance based on the Smallest Enclosing Ball.}

3.1. The Smallest Enclosing Ball. Let $(X, d)$ be a non trivial $(|X| \geqslant 2)$ proper metric space.

Proposition 1. Given a list $\left(x_{1}, \ldots, x_{n}\right) \in X^{n}$, let us consider the function $F\left(x_{1}, \ldots, x_{n}\right): X \rightarrow R^{+}$defined by

$$
F_{\left(x_{1}, \ldots, x_{n}\right)}(p)=\max \left\{d\left(p, x_{i}\right), i=1, \ldots, n\right\}=\bigvee_{i=1}^{n} d\left(p, x_{i}\right)
$$

Then $F$ satisfies the following properties:

i) $F_{\left(x_{1}\right)}(p)=d\left(p, x_{1}\right)$

ii) $F_{\left(x_{1}, x_{2}\right)}(p) \geq \frac{1}{2} d\left(x_{1}, x_{2}\right)$

iii) $F_{\left(x_{1}, \ldots, x_{n}\right)}$ is a continuous function and it achieves the absolute minimum.

Proof. The first property comes from the definition of $F$. Let us prove ii). First of all, note that for all $p \in X$ we have

$$
d\left(x_{1}, x_{2}\right) \leq d\left(x_{1}, p\right)+d\left(x_{2}, p\right) \leq 2 \max \left\{d\left(p, x_{1}\right), d\left(p, x_{2}\right)\right\}
$$

Thus $F_{\left(x_{1}, x_{2}\right)}(p)=\max \left\{d\left(p, x_{1}\right), d\left(p, x_{2}\right)\right\} \geq \frac{1}{2} d\left(x_{1}, x_{2}\right)$

Finally we prove iii). Let $f_{i}: X \rightarrow R^{+}$be defined by $f_{i}(p)=d\left(p, x_{i}\right), i=1, \ldots, n$. All theses functions are continuous since $\left|d\left(p, x_{i}\right)-d\left(p \prime, x_{i}\right)\right| \leq d(p, p \prime)$ for $i=1, \ldots n$.

Now $F_{\left(x_{1}, \ldots, x_{n}\right)}=\bigvee_{i=1}^{n} f_{i}$, and thus $F_{\left(x_{1}, \ldots, x_{n}\right)}$ is continuous. 
For the sake of simplicity, we will write $F$ instead of $F_{\left(x_{1}, \ldots, x_{n}\right)}$. Let us prove now that $F$ achieves the absolute minimum.

There exists a closed ball $B$ containing $x_{1}, \ldots, x_{n}$ and a point $p^{\prime} \in B$ such that $F\left(p^{\prime}\right) \leq F(p) \forall p \in B^{c}$.

Since $F$ is continuous and $B$ a compact set, then $F$ achieves a minimum on B:

$$
\exists p_{0} \in B \quad / \quad F\left(p_{0}\right) \leq F(p) \forall p \in B
$$

But also $F\left(p_{0}\right) \leq F\left(p^{\prime}\right) \leq F(p) \forall p \in B^{c}$. Thus $F\left(p_{0}\right) \leq F(p) \forall p \in X$.

REMARK 2. If the metric space $X$ is not proper, the function $F_{\left(x_{1}, \ldots, x_{n}\right)}$ needs not have a minimum. For instance, the set $X=Q \cup\{\sqrt{2}\}$ with the euclidean distance is not a proper metric space. In effect, the closed ball $B(0 ; \sqrt{2})=\{x \in Q \cup\{\sqrt{2}\} ;|x| \leq$ $\sqrt{2}\}$ is not compact since it is not a closed set.

Now, let us consider the function $F_{(0, \sqrt{2})}$. For all $p \in X$,

$$
F_{(0, \sqrt{2})}(p)=\max \left\{d(p, 0), d(p, \sqrt{2}\}=\left\{\begin{array}{cl}
\sqrt{2}-p & \text { if } p<\frac{\sqrt{2}}{2} \\
p & \text { if } p>\frac{\sqrt{2}}{2}
\end{array}\right.\right.
$$

Then this function does not have a minimum.

Proposition 2. The radius of the smallest closed ball containing the points $x_{1}, \ldots, x_{n} \in X$ is the real number

$$
\min \left\{\max \left\{d\left(p, x_{i}\right) ; i=1 \ldots, n\right\} ; p \in X\right\}
$$

Proof. In effect, let $p_{0} \in X$ be such that

$$
r=\min \left\{\max \left\{d\left(p, x_{i}\right) ; i=1 \ldots, n\right\}, p \in X\right\}=\max \left\{d\left(p_{0}, x_{i}\right) ; i=1 \ldots, n\right\}
$$

The closed ball $B\left(p_{0} ; r\right)$ contains the points $x_{1}, \ldots, x_{n}$, that is, $d\left(p_{0}, x_{i}\right) \leq r$.

If $B\left(p_{1} ; r_{1}\right)$ is another closed ball containing the points $x_{1}, \ldots, x_{n}$, then $d\left(p_{1}, x_{i}\right) \leq$ $r_{1}$ for all $i=1 \ldots, n$, and $\max \left\{d\left(p_{1}, x_{i}\right) ; i=1 \ldots, n\right\} \leq r_{1}$. Therefore $r \leq r_{1}$.

Next we recall a well known result about the uniqueness of the smallest enclosing ball in the euclidean space. We include the proof to emphasize the importance of the midpoint property to ensure this uniqueness.

Proposition 3. Let us consider the euclidean space $R^{m}$. Then there exists the smallest enclosing ball of a finite set of points and it is unique.

Proof. Let $P$ be a finite set of points of $R^{m}$. The smallest enclosing ball containing $P$ exists since $R^{m}$ is a proper metric space.

In order to prove the uniqueness, let us suppose that there exist two closed balls $B_{1}$ and $B_{2}$ containing $P$, with centers $c_{1}$ and $c_{2}$, respectively, and minimum radius $r$. Then $P \subset B_{1} \cap B_{2}$ and $B_{1} \cap B_{2}$ is contained in a ball with center the midpoint of $c_{1}$ and $c_{2}, c=\frac{1}{2}\left(c_{1}+c_{2}\right)$, and radius $r^{\prime}=\sqrt{r^{2}-a^{2}}$ where $a=\frac{1}{2} d\left(c_{1}, c_{2}\right)$. 
In effect, if $a>0$ and $d\left(p, c_{1}\right) \leq r$ and $d\left(p, c_{2}\right) \leq r$, then if we apply the Apollonius' Theorem to the triangle $p, c_{1}, c_{2}$, we have

$$
d(p, c)=\frac{1}{2} \sqrt{2\left(d\left(p, c_{1}\right)^{2}+d\left(p, c_{2}\right)^{2}\right)-d\left(c_{1}, c_{2}\right)^{2}} \leq \sqrt{r^{2}-a^{2}}=r^{\prime}
$$

Thus $B\left(c ; r^{\prime}\right)$ is a ball containing the set $P$ and its radius $r^{\prime}<r$, but this it is not possible, therefore $a=0$ and $c_{1}=c_{2}$.

REMARK 3. The midpoint property (see Def. 4 below) in a metric space does not imply the uniqueness of the ball of minimum radius containing several points. For instance, the euclidean plane $R^{2}$, with the distance of the maximum, that is,

$$
d\left(\left(x_{1}, x_{2}\right),\left(y_{1}, y_{2}\right)\right)=\max \left\{\left|x_{1}-y_{1}\right|,\left|x_{2}-y_{2}\right|\right\}
$$

satisfies the midpoint property but the smallest ball is not unique: if we take the points $(0,0)$ and $(1,0)$, all the squares of side 1 with these two points in there border would be smallest balls. The uniqueness would hold only in particular cases, like for example when the points are opposing vertices of a square.

3.2. Smallest Enclosing Ball Multidistance. In this section we analyze in which conditions a multidimensional function $D\left(x_{1}, \ldots, x_{n}\right)$ defined as the diameter of the smallest enclosing ball of $x_{1}, \ldots, x_{n}$ is a multidistance.

Definition 3. We say that the metric space $(X, d)$ has the Fermat property ( $X$ is a Fermat space) if for all $x_{1}, \ldots, x_{n} \in X^{n}$, there exists a ball of radius $\frac{F}{2}$ containing the points $x_{1}, \ldots, x_{n}$, where

$$
F=\min \left\{\sum_{i=1}^{n} d\left(p, x_{i}\right) ; p \in X\right\}
$$

REMARK 4. $F$ is called the Fermat sum and a point $f$ where this minimum value is reached is called a Fermat point.

Let $x_{1}, \ldots, x_{n}, x_{n+1} \in X$ and let $F_{n}$ and $F_{n+1}$ be the Fermat sums of $x_{1}, \ldots, x_{n}$ and $x_{1}, \ldots, x_{n}, x_{n+1}$, respectively. Then $F_{n} \leq F_{n+1}$. In effect, let $f_{n}$ and $f_{n+1}$ be two corresponding Fermat points. Then

$$
F_{n}=\sum_{i=1}^{n} d\left(f_{n}, x_{i}\right) \leq \sum_{i=1}^{n} d\left(f_{n+1}, x_{i}\right) \leq \sum_{i=1}^{n+1} d\left(f_{n+1}, x_{i}\right)=F_{n+1}
$$

EXAMPLE 2. Let us give an example of a proper metric space not having the Fermat property.

Let us consider $X=[a, b] \cup[c, e] \subset R$ where $a<b<c<e$, with the usual distance. This is a proper space but it does not have the Fermat property: the points $b, c$ cannot be included in any ball with radius $F / 2$, where

$$
F=\min \{d(p, b)+d(p, c) ; p \in[a, b] \cup[c, e]\}=c-b
$$


DeFINITION 4. We say that the metric space $(X, d)$ has the midpoint property if for each $x, y \in X$ there exists $m \in X$ such that

$$
d(x, y)=2 d(m, x)=2 d(m, y)
$$

Proposition 4. If $(X, d)$ has the Fermat property, then it has the midpoint property.

Proof. Let us consider two points $x, y \in X$ and let

$$
F=\min \{d(p, x)+d(p, y) ; p \in X\}
$$

Since $X$ has the Fermat property, then we know that there exists $m \in X$ such that $d(m, x) \leq \frac{F}{2}$ and $d(m, y) \leq \frac{F}{2}$. But then $F \leq d(m, x)+d(m, y) \leq F$ and therefore $d(m, x)=d(m, y)=\frac{F}{2}$.

On the other hand, $d(x, y) \leq d(x, m)+d(m, y)=F$ but also $d(x, y) \geq F$, from the definition of $F$. Therefore, $d(x, y)=2 d(m, x)=2 d(m, y)$, and $m$ is a midpoint between $x$ and $y$.

Next we introduce a multidimensional function $D\left(x_{1}, \ldots, x_{n}\right)$ corresponding to the diameter of the smallest enclosing ball of the list.

Definition 5. Let us define $D: \bigcup_{n \geqslant 1} X^{n} \rightarrow R^{+}$in the following way:

$$
D\left(x_{1}, \ldots, x_{n}\right)=\min \left\{2 \max \left\{d\left(p, x_{i}\right) ; \quad i=1, \ldots, n\right\} ; p \in X\right\}=2 \bigwedge_{p \in X} \bigvee_{i=1}^{n} d\left(p, x_{i}\right)
$$

Proposition 5. The restriction of $D$ to $X^{2}$ is $d$ ( $D$ is an extension of $\left.d\right)$ if and only if $(X, d)$ has the midpoint property.

Proof. Supose first that $X$ has the midpoint property and let $x_{1}, x_{2} \in X$. For all $p \in X$, we have that

$$
d\left(x_{1}, x_{2}\right) \leq d\left(x_{1}, p\right)+d\left(x_{2}, p\right) \leq 2 \max \left\{d\left(x_{1}, p\right), d\left(x_{2}, p\right)\right\}
$$

Therefore

$$
D\left(x_{1}, x_{2}\right)=\min \left\{2 \max \left\{d\left(p, x_{1}\right), d\left(p, x_{2}\right)\right\}, p \in X\right\} \geq d\left(x_{1}, x_{2}\right)
$$

On the other hand, if $m$ is a midpoint between $x_{1}$ and $x_{2}$, we have

$$
d\left(x_{1}, x_{2}\right)=2 d\left(m, x_{1}\right)=2 d\left(m, x_{2}\right)
$$

and $\frac{1}{2} d\left(x_{1}, x_{2}\right)$ is an element of the set $\left\{\max \left\{d\left(p, x_{1}\right), d\left(p, x_{2}\right)\right\} ; p \in X\right\}$; thus

$$
D\left(x_{1}, x_{2}\right)=\min \left\{2 \max \left\{d\left(p, x_{i}\right) ; \quad i=1, \ldots, n\right\} ; p \in X\right\} \leq d\left(x_{1}, x_{2}\right)
$$

and $D\left(x_{1}, x_{2}\right)=d\left(x_{1}, x_{2}\right) \forall x_{1}, x_{2} \in X$. 
Let us suppose now that $D\left(x_{1}, x_{2}\right)=d\left(x_{1}, x_{2}\right) \forall x_{1}, x_{2} \in X$, that is,

$$
\min \left\{2 \max \left\{d\left(p, x_{1}\right), d\left(p, x_{2}\right)\right\} ; p \in X\right\}=d\left(x_{1}, x_{2}\right)
$$

This means that there exists $m \in X$ such that

$$
2 \max \left\{d\left(m, x_{1}\right), d\left(m, x_{2}\right)\right\}=d\left(x_{1}, x_{2}\right)
$$

and then

$$
d\left(x_{1}, x_{2}\right)=2 d\left(m, x_{1}\right)=2 d\left(m, x_{2}\right)
$$

Thus $m$ is a midpoint between $x_{1}$ and $x_{2}$.

Proposition 6. Let $(X, d)$ be a proper metric space. Then the function $D$ : $\bigcup_{n \geqslant 1} X^{n} \rightarrow R^{+}$defined by

$$
D\left(x_{1}, \ldots, x_{n}\right)=2 \bigwedge_{p \in X} \bigvee_{i=1}^{n} d\left(p, x_{i}\right)
$$

is a multidistance extension of $d$ if and only if $X$ has the Fermat property.

Proof. Let us suppose first that $D$ is a multidistance extension of $d$ and let us prove that $X$ has the Fermat property.

From the condition (3) of the definition of multidistance, we have that

$$
D\left(x_{1}, \ldots, x_{n}\right)=2 \bigwedge_{p \in X} \bigvee_{i=1}^{n} d\left(p, x_{i}\right) \leq \sum_{i=1}^{n} d\left(x_{i}, y\right) \forall y \in X
$$

Thus there must exist $p_{0} \in X$ such that for all $i=1, \ldots, n$,

$$
d\left(p_{0}, x_{i}\right) \leq \frac{1}{2} \sum_{i=1}^{n} d\left(x_{i}, y\right) \forall y \in X
$$

But then

$$
d\left(p_{0}, x_{i}\right) \leq \frac{F}{2} \forall i=1, \ldots, n
$$

and $X$ has the Fermat property.

Reciprocally, let us suppose that $X$ has the Fermat property. First of all, we have from the propositions 4 and 5 that $D$ is an extension of $d$. Let us now prove that $D$ is a multidistance, that is, for all $n \geq 1$ and $x_{1}, \ldots, x_{n}, y \in X$ :

(1) $D\left(x_{1}, \ldots, x_{n}\right)=0$ if and only if $x_{i}=x_{j}$ for all $i, j=1, \ldots n$,

(2) $D\left(x_{1}, \ldots, x_{n}\right)=D\left(x_{\pi(1)}, \ldots, x_{\pi(n)}\right)$ for any permutation $\pi$ of $1, \ldots, n$,

(3) $D\left(x_{1}, \ldots, x_{n}\right) \leqslant D\left(x_{1}, y\right)+\ldots+D\left(x_{n}, y\right)$. 
The condition (1) comes from the fact that $D\left(x_{1}, \ldots, x_{n}\right)$ is the diameter of the smallest ball containing the points $x_{1}, \ldots, x_{n}$, and (2) is obvious from the definition of $D$. Now, we have to prove (3), that is,

$$
2 \bigwedge_{p \in X} \bigvee_{i=1}^{n} d\left(p, x_{i}\right) \leqslant d\left(x_{1}, y\right)+\ldots+d\left(x_{n}, y\right) \forall y \in X
$$

This is equivalent to prove that for all $y \in X$, there exists $p \in X$ such that

$$
\max \left\{d\left(p, x_{i}\right) ; i=1, \ldots, n\right\} \leqslant \frac{1}{2} \sum_{i=1}^{n} d\left(x_{i}, y\right)
$$

Let $p_{0}$ be a point of $X$ such that $d\left(p_{0}, x_{i}\right) \leq \frac{F}{2} \forall i=1, \ldots, n$, and the proof is finished since $F \leq \sum_{i=1}^{n} d\left(x_{i}, y\right) \forall y \in X$.

Next example shows that $D\left(x_{1}, \ldots, x_{n}\right)=2 \bigwedge_{p \in X} \bigvee_{i=1}^{n} d\left(p, x_{i}\right)$ could be a multidistance even if $X$ is not a Fermat space.

EXAMPLE 3. Let $X$ be a finite set with $|X| \geq 2$ and let $d$ be the drastic distance on $X . X$ is a proper metric space that does not have the midpoint property and thus it is not a Fermat space. In this case,

$$
D\left(x_{1}, \ldots, x_{n}\right)= \begin{cases}0 & \text { if } x_{i}=x_{j} \\ 2 & \text { otherwise }\end{cases}
$$

is a multidistance with $D\left(x_{1}, x_{2}\right)=2 d\left(x_{1}, x_{2}\right)$.

Note that $\frac{1}{2} D\left(x_{1}, \ldots, x_{n}\right)$ is the multidimensional version of the discrete distance.

Proposition 7. The euclidean space $R^{m}, m \geq 1$, is a Fermat space.

Proof. Let us take $x_{1}, \ldots, x_{n} \in R^{m}$. Let us suppose that $x_{1}$ and $x_{2}$ are the most distant points of the list and let $m$ be their midpoint. Let us suppose now that $x_{3}$ satisfies that $d\left(m, x_{3}\right) \geq d\left(m, x_{i}\right) \forall i=3, \ldots, n$. Then any ball with center at $m$ containing $x_{1}, x_{2}$ and $x_{3}$ must contain also $x_{4}, \ldots, x_{n}$. Thus if we consider the plane defined by $x_{1}, x_{2}$ and $x_{3}$, we can reduce the problem to the case of three points in $R^{2}$.

Let us consider then $A, B, C \in R^{2}$ and suppose that $d(B, C) \geq d(A, B) \vee d(A, C)$. Let $a, b, c$ be the lengths of the segments $B C, A C$ and $A B$, respectively. Let $M$ be the midpoint of the segment $B C$ and let $d=d(M, A)$.

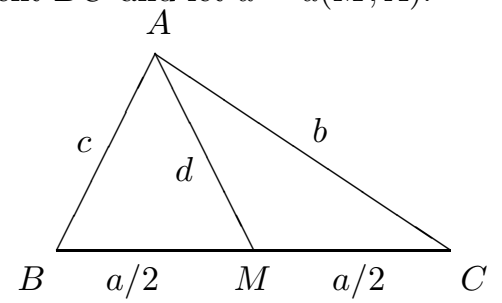

FIG. 1. The triangle $A B C$. 
In order to prove the Fermat property, it is sufficient to prove that $d \leq F / 2$, where

$$
F=\min \left\{d(P, A)+d(P, B)+d(P, C) ; P \in R^{2}\right\}
$$

Observe first that $a \leq F$ and thus, if $A \in B(M ; a / 2)$, then $A \in B(M ; F / 2)$ and the proof is finished. Let us suppose now that $A \notin B(M ; F / 2)$. Since we must have that $A \in B(B ; a) \cap B(C ; a), A B C$ must be an acute triangle.

Now, by applying the Law of cosines to both the triangle $A B M$ with sides $a / 2, c$ and $d$ and the triangle $A B C$ with sides $a, b$ and $c$, we have

$$
d^{2}=\frac{a^{2}}{4}+c^{2}-2 \cdot \frac{a}{2} \cdot c \cdot \cos B=\frac{a^{2}}{4}+c^{2}-a c \cdot \frac{a^{2}+c^{2}-b^{2}}{2 a c}=\frac{b^{2}}{2}+\frac{c^{2}}{2}-\frac{a^{2}}{4}
$$

On the other hand, a straightforward application of the Law of cosines and Heron's formula shows that the Fermat sum of a triangle is equal to

$$
F=\frac{\sqrt{2}}{2} \sqrt{a^{2}+b^{2}+c^{2}+\sqrt{3} \cdot \sqrt{\left((b+c)^{2}-a^{2}\right)\left(a^{2}-(b-c)^{2}\right)}}
$$

Thus a straightforward computation gives

$$
\begin{aligned}
\left(\frac{F}{2}\right)^{2} & =\frac{1}{8}\left(a^{2}+b^{2}+c^{2}+\sqrt{3} \cdot \sqrt{\left((b+c)^{2}-a^{2}\right)\left(a^{2}-(b-c)^{2}\right)}\right) \\
& =\frac{1}{8}\left(a^{2}+b^{2}+c^{2}+\sqrt{3} \cdot \sqrt{2 a^{2} b^{2}+2 a^{2} c^{2}+2 b^{2} c^{2}-a^{4}-b^{4}-c^{4}}\right)
\end{aligned}
$$

Now, taking into account that the angle $A$ is acute and thus $\cos A>0$, which implies that $a^{2}=b^{2}+c^{2}-2 b c \cos A<b^{2}+c^{2}$, we have

$$
\begin{aligned}
d^{2} \stackrel{?}{\leq} \frac{F}{2} & \Longleftrightarrow 3 b^{2}+3 c^{2}-3 a^{2} \stackrel{?}{\leq} \sqrt{3} \cdot \sqrt{2 a^{2} b^{2}+2 a^{2} c^{2}+2 b^{2} c^{2}-a^{4}-b^{4}-c^{4}} \\
& \Longleftrightarrow a^{4}+b^{4}+c^{4}+b^{2} c^{2}-2 a^{2} b^{2}-2 a^{2} c^{2} \stackrel{?}{\leq} 0 \\
& \Longleftrightarrow\left(a^{2}-b^{2}\right)^{2} \stackrel{?}{\leq} c^{2}\left(2 a^{2}-b^{2}-c^{2}\right)
\end{aligned}
$$

And this inequality is true:

$\left(a^{2}-b^{2}\right)^{2}=\left(a^{2}-b^{2}\right)\left(a^{2}-b^{2}\right)=\left(c^{2}-2 b c \cos A\right)\left(a^{2}-b^{2}+a^{2}-c^{2}\right) \leq c^{2}\left(2 a^{2}-b^{2}-c^{2}\right)$

since

- the angle $A$ is acute, thus $\cos A>0$

. $a>c \Longrightarrow a^{2}-c^{2}>0$

. $a>b \Longrightarrow a^{2}>b^{2}$ 
4. Conclusions. In this paper, we prove that, given a finite set of points $P$ of a proper metric space, there exists the smallest closed ball containing $P$. This ball is proven to be unique for the case of $R^{m}$. On the other hand, we consider the diameter of the smallest enclosing ball of a set of points $\left\{x_{1}, \ldots, x_{n}\right\}$ in a proper metric space $X$ as a multi-argument function $D\left(x_{1}, \ldots, x_{n}\right)$, and analyse under which conditions this measure is, in fact, a multidistance. More precisely, we prove that $D$ is a multidistance if, and only if, $X$ has the Fermat property. In particular, we prove that this is always true for the Euclidean space $R^{m}$.

Acknowledgment. The authors acknowledge the support of the Spanish DGI grant MTM2009-10962.

\section{REFERENCES}

[1] T. Calvo, J. Martín, G. Mayor, Measures of desagreement and aggregation of preferences based on multidistances. Proceedings of the IPMU 2012, Part IV, pp. 549-558.

[2] D. Cheng, X. Hu, C. Martin, On the Smallest Enclosing Balls, Communications in Information and Systems, 6:2(2006), pp. 137-160.

[3] J. Martín, G., Mayor.Aggregating pairwise distance values, Proceedings of the EUROFUSE 2009, pp. 147-152.

[4] J. Martín, G. Mayor, Multi-argument Distances, Fuzzy Sets and Systems, 167(2011), pp.92100 .

[5] N. Megiddo, Linear Programming in Linear Time when the Dimension is Fixed, J. ACM, 31:6(1984), pp. 114-127.

[6] B. Mordukhovich, N. Mau Nam, C. Villalobos, The Smallest Enclosing Ball Problem and the Smallest Intersecting Ball Problem: Existence and Uniqueness of Solutions, Optimization Letters, 7:5(2013), pp. 839-853.

[7] J.J. Sylvester, A question in the Geometry of Situation, Quaterly Journal of Pure and Applied Mathematics, 1:79(1857).

[8] D.H. Wolpert, Metrics for more than two points at once, International Conference on Complex Systems ICCS 2004, Y. Bar-Yam (Ed.), Perseus Books. 